\section{PS48 PROCESSES OF CHANGE: WHAT ARE THE SPECIFIC PATHWAYS AND PROJECT COMPONENTS THAT HAVE A POSITIVE IMPACT ON THE WELL-BEING OF PARTICIPANTS IN A 'WELL LONDON' PROJECT?}

doi:10.1136/jech-2012-201753.147

1J Derges, 'A Draper, ${ }^{2} \mathrm{~A}$ Clow, ${ }^{1} \mathrm{R}$ Lynch, ${ }^{1} \mathrm{~S}$ Jain. ${ }^{1}$ School of Life Sciences, University of Westminster, London, UK; ${ }^{2}$ Psychology Department, University of Westminster, London, UK

Background This paper presents follow-up data from a qualitative study of a Big Lottery funded 'Well London' project; an initiative set up to improve the health and well-being of Londoners living in areas historically marked by social, economic and environmental deprivation. The project introduced a series of health interventions: healthy eating, physical activity, and mental health and well-being, across a total of 20 areas within London. An evaluation of the interventions was conducted through a series of in depth semi-structured interviews with residents in 3 of the areas. Participants were interviewed twice: initially at the start of the interventions and again twelve months later. The interviews explored which of the project components acted positively on participant's sense of well-being and lead to changes in health behaviour. The methodology used, placed emphasis on the opinions and reflections of study participants and responds to Popay's call for research to be centred not only on theoretical concerns, but also to be attentive to 'lay' theories of health. Methods Two sets of 60 semi-structured interviews, taking place a year apart were conducted across three locations identified as Census Lower Super Output Areas (LSOAs). These measured changes in health behaviour and residents' perceptions of 'well-being' that arose following the Well London interventions. Residents were interviewed about their experiences and perceptions of the neighbourhood; also their health and views of the Well London interventions. Residents who had not taken part in the project interventions were also selected and interviewed, for comparison.

Results The study found that the project objectives succeeded where there were at least one of the following present; a) high levels of individual motivation; b) the involvement of external agencies in the form of key worker and/or agency support; c) where residents were involved in the organisation and dissemination of the projects, often as volunteers; and d) where the environment was made accessible, safe and conducive to a sense of belonging and 'community ownership'.

Conclusion The findings indicate that the Well London project increased perceptions of well-being in populations defined by inner city social disadvantage. However, the pathways to change are complex and not easily generalized. Success is dependent on multiple interacting interpersonal, social and contextual factors, as well as the actual type of interventions themselves.

\section{PS49 FOOD FOR THOUGHT! THE ROLE OF DIETARY CHOICES ON COGNITIVE BEHAVIOUR}

doi:10.1136/jech-2012-201753.148

1.2D Cadar, ${ }^{2 H}$ Pikhart, ${ }^{3} \mathrm{G}$ Mishra, ${ }^{4} \mathrm{AM}$ Stephen, ${ }^{1} \mathrm{D}$ Kuh, ${ }^{1} \mathrm{M}$ Richards. ${ }^{1} \mathrm{M} R \mathrm{C}$ Unit for Lifelong Health and Ageing, UCL, London, UK; 'Institute of Epidemiology and Health Care, UCL, London, UK; ${ }^{3}$ School of Population Health, University of Queensland, Herston, Australia; ${ }^{4}$ MRC Human Nutrition Research, University of Cambridge, Cambridge, UK

Background Cognitive decline indicates the first sign of dementia and a major public health impact associated with ageing. Evidence suggests that dietary patterns are associated with cerebrovascular and cardiovascular risk factors.

Methods To examine the association between dietary choices and 20 years cognitive decline from 43 to 60+.; 1018 study members from the MRC National Survey of Health and Development regularly followed up since birth in 1946 were included in the study.
Cognitive functioning was measured at 43 and 60+ years using two tests: verbal memory (maximum words recalled 45 ) and visual search (maximum letter searched: 600).

Dietary data was obtained using a 5 days diet diary at age 36 and $43 y$. An overall score representing level of healthy food choice was derived, by summing scores from five separate criteria: 1. consumption of breakfast ( 0 no consumption to 2 all days); 2.type of milk (from 0 whole to 3 skim milk); 3 . type of bread (from 0 white to 4 wholemeal); 4. number of daily portions of fruit and vegetables (from 0 none to 5 portions/day) and percentages of energy from daily intakes (0-more than $45 \%$ energy to 5 -less than 30\% energy). A total score was derived and further classified as 0 low or 1 higher choice, subject to median split at each age. A cumulative midlife score was further derived as 0 low choice at either age or 1 higher choice at least at one age or both. The confounding variables were father's social class, childhood cognition, education, adult social class and depression.

Results There was a cross sectional positive association between a higher choice of diet and verbal memory scores at age $43 y$ compared to a lower choice 1.83 (95\% CI 1.12 to 2.54 ). This association was maintained after adjusting for all covariates. There was also a significant association between a midlife cumulative healthier choice of diet and a slower verbal memory from 43 to $60+$ in a fully adjusted model 0.69 (1.10 to 1.27) compared to midlife lower choice. There was no association between dietary choice and visual search or visual search decline.

Conclusion Our results suggest that a healthy dietary option based on high intake of fruit and vegetables, complex carbohydrates and cereals made in early midlife is protective of verbal memory decline from mid to later life. Public health interventions based on healthy diets and the prevention of nutritional deficiencies should be considered an important line of defence against cognitive decline and dementia.

\section{PS50 THE DEVELOPMENT OF MINIMUM UNIT PRICING OF ALCOHOL IN SCOTLAND: A POLICY CASE STUDY}

doi:10.1136/jech-2012-201753.149

${ }^{1}$ SV Katikireddi, ${ }^{2}$ S Hilton, 'L Bond. 'Evaluating the Health Effects of Social Interventions programme, MRC/CSO Social and Public Health Sciences Unit, Glasgow, UK; ${ }^{2}$ Understanding and Uses of Public Health Research, MRC/CSO Social and Public Health Sciences Unit, Glasgow, UK

Background Awareness of alcohol as a public health issue has increased in the UK and elsewhere over recent years. Evidence exists to support the use of price mechanisms to address alcohol harms. While various Canadian provinces have used reference pricing to control off-license alcohol prices in state-run monopolies, no country has implemented a uniform minimum unit price (MUP) that applies to all alcohol sales. This policy is being actively considered in Scotland, with legislation likely to be passed this year. Studying developments in Scotland may therefore help those working in public health to improve their engagement with policymakers as well as being of use to other countries seeking to introduce MUP in the future.

Methods Political science theories can be used to identify potential explanations for the development of policy. We draw on a number of theories to allow a range of explanations to be identified (a 'multiple-lenses' approach). Punctuated-Equilibrium Theory (PET) suggests policymakers use their limited time to address a few issues in detail (which can experience major policy change), while paying little attention to most other policy areas. Alternatively, multi-level governance highlights the diversity of actors seeking to influence policy within different governmental levels (venues). The same policy issue can therefore be considered at European Union, Westminster Parliament or Scottish Parliament, for example. We utilise political science theories to provide explanations for the 
development of minimum unit pricing for alcohol as a public health policy in Scotland.

Results PET highlights the importance of redefining the policy problem so that it becomes a focus for policymaking. This therefore suggests reframing of alcohol as a public health problem amenable to a population-based solution has been instrumental in bringing about consideration of MUP. A multi-level governance framework suggests that the devolution of health (but not trade or taxation policy) to Scottish Parliament illustrates how public health advocates were able to 'venue shop' from Westminster to the Scottish Parliament. We also provide a necessary description of the process through which MUP has emerged.

Conclusion Political science theories are useful for understanding public health policy developments and could be more widely used by the public health community to inform advocacy or engagement with policymakers. This case study illustrates their value as well as providing more generalisable lessons for public health advocates (such as to seek opportunities for 'venue shopping' and an appreciation of the importance of framing of policy issues).

\section{PS51 COULD MORE THAN THREE MILLION OLDER PEOPLE BE AT RISK OF ALCOHOL-RELATED HARM? A CROSS. SECTIONAL ANALYSIS OF AGE-SPECIFIC DRINKING GUIDELINES}

doi:10.1136/jech-2012-201753.150

C Knott, S Scholes, NJ Shelton. Epidemiology and Public Health, UCL, London, UK

Background To determine the effect of recently proposed age-specific alcohol consumption guidelines upon the estimated proportion and number of older individuals potentially at risk of alcohol-related harm. Nationally representative cross-sectional population data from Health Survey for England (HSE).

Methods Random sample of the general population living in private households in England. For reliable comparison of adult alcohol consumption by age and sex across a five-year period, data were extracted from HSE 2003 and 2008 for all participants aged 16 or over. The sample included those who drank in the previous week, never drinkers, non-drinkers and adults who reported not having consumed alcohol in the week prior to interview. Excluded from the sample were those for whom data on drinking behaviour or unit alcohol consumption were missing (around one per cent of the total available sample). The sample from HSE 2003 thus comprised 14,718 participants, while the 2008 sample comprised data on 14,939 individuals.

Results The total number of individuals aged 65 or over classified as drinking in excess of daily recommended limits would have increased to over three million in 2008 under age-specific guidelines proposed by the Royal College of Psychiatrists, equating to an at-risk population of 809,000 individuals greater than found within the 16-24 age group during the same year. Proposed revisions to existing binge drinking classifications defined almost $1,200,000$ people aged 65 or over as hazardous consumers of alcohol in 2008 , equating to a rise of $258 \%$ by comparison with existing definitions.

Conclusion The introduction of new age-specific drinking guidelines put forward by the Royal College of Psychiatrists would increase the elderly population drinking in excess of daily recommended limits by more than two-fold, and increase by more than three-fold the number of binge drinkers. However, there remains at present a dearth of evidence sufficient for guiding age-specific revisions to existing and already problematic alcohol consumption thresholds. Nevertheless, vigilance regarding heavy late-life drinking remains important in light of older people's heightened sensitivity to the effects of alcohol and the rising number within the sub-population potentially at risk of alcoholrelated harm.

\section{PS52 ARE THERE GENDER DIFFERENCES IN THE SOCIAL PATTERNING OF BINGE DRINKING IN THE CZECH REPUBLIC, RUSSIA, POLAND AND LITHUANIA? A CROSS- SECTIONAL STUDY}

doi:10.1136/jech-2012-201753.151

L Asher, H Pikhart, M Bobak. Epidemiology and Public Health, UCL, London, UK

Background Binge drinking may have played a central role in mortality fluctuations in the former Soviet Union (fSU). In Eastern Europe, binge drinking is more common in men than women and in men of lower socio-economic position (SEP). Yet intersections of these factors have rarely been addressed with sufficient power to detect social patterns in binge drinking amongst women. The aim of this study was to determine whether there are gender differences in the social patterning of binge drinking in the Czech Republic, Russia, Poland and Lithuania.

Methods Cross-sectional baseline data from the HAPIEE (Health, Alcohol and Psychosocial factors in Eastern Europe) study was used. The participants were men and women aged $45-69$ years randomly selected from population registers in the Czech Republic, Russia, Poland and Lithuania. Logistic regression was used to examine the association between social factors (education, employment status, household amenities and deprivation) and binge drinking (>100g (men) and $>60 \mathrm{~g}$ (women) ethanol per occasion $\geq 1 \mathrm{x} /$ month) in each gender and country separately. Amenities score was derived from 18 (Lithuania) or 12 (other countries) individual questions on household items. 'Few amenities' was defined as a score in the lowest quartile for the participant's country.

Tests for interaction between gender and each variable were carried out. Tests for interaction between country and each variable were also completed. Pooled data (all countries) was interpreted where there was no evidence of country heterogeneity.

Results There were 34,069 participants with complete data. Amongst men in all countries, all markers of low SEP were associated with increased odds of binge drinking (e.g. OR $1.22,95 \% \mathrm{CI}$ 1.10-1.35 for few amenities, fully adjusted model). In women, few amenities (OR 0.81, 0.70-0.94, fully adjusted model), and to a lesser extent low education level, were associated with decreased odds of binge drinking. The associations between social factors, gender and binge drinking were homogenous between countries (few amenities test for country heterogeneity $p=0.72$ men, $p=0.51$ women) with the exceptions of education and deprivation in women.

Conclusion In Russia and Eastern Europe binge drinking in men and women is socially patterned, but in contrasting ways. Men with lower SEP may binge drink due to social instability and poor coping mechanisms. Women with higher SEP may binge drink due to greater resources and exposure to, and adoption of, male drinking patterns through employment or higher education. Policy to tackle binge drinking should take into account its gender-specific social determinants.

\section{PS53 EFFECT OF GEOGRAPHICAL ACCESS TO HEALTH FACILITIES ON CHILD MORTALITY IN RURAL ETHIOPIA: A COMMUNITY BASED CROSS SECTIONAL STUDY}

doi:10.1136/jech-2012-201753.152

${ }^{1} Y$ Okwaraji, ${ }^{\prime} \mathrm{C}$ Cousens, ${ }^{2} \mathrm{Y}$ Berhane, ${ }^{1} \mathrm{~K}$ Mulholland, ${ }^{1} \mathrm{~K}$ Edmond. ${ }^{1}$ Epidemiology \& Population Health, LSHTM, London, UK; ${ }^{2}$ Epidemiology \& Biostatistics, Addis Ababa University, Addis Ababa, Ethiopia

Background There have been few studies which have examined associations between access to health care and child health outcomes in remote populations most in need of health services. This study assessed the effect of travel time and distance to health facilities on mortality in children under five years in a remote area of rural north-western Ethiopia. 